\title{
Índice de aceites residuales de cocina para la producción de biodiésel en las provincias de Lima y Trujillo. 2020
}

\author{
Jose Alfredo Zarate Gamarra, Bachiller en la carrera de Ingeniería Ambiental ${ }^{1}$, Jessica Marleny Luján Rojas, \\ Magister en Ciencias², Grant Ilich LLaque Fernández, Magister en Educación ${ }^{3}$ \\ ${ }^{1}$ Universidad Privada del Norte, Perú, joseskekun@gmail.com \\ ${ }^{2}$ Universidad Privada del Norte, Perú, jessica.lujan@upn.pe \\ ${ }^{3}$ Universidad Privada del Norte, Perú, grant.llaque@upn.pe
}

\begin{abstract}
Resumen- La continua contaminación ha generado la búsqueda de energías renovables, teniendo a los biocombustibles como respuestas, especialmente al biodiésel a partir de aceite residual, que utiliza un desecho que no compite con el sector alimenticio. La investigación tuvo como objetivo analizar el índice de aceites residuales de cocina para la producción de biodiésel en las provincias de Lima y Trujillo. Recolectándose información de trabajos de investigación, obteniendo los parámetros como densidad, viscosidad e índice de acidez, comparando la dispersión del aceite residual y el biodiésel mediante diagramas de cajas. De igual forma, se contrastaron los catalizadores y alcoholes empleados con los parámetros, agregándose el rendimiento. Adicionalmente, se realizó una comparación de la normativa del aceite vegetal peruano a nivel internacional, así como, las metodologías de las investigaciones. Para finalizar, se propone el diseño de una planta de producción de biodiésel a partir de aceite vegetal reciclado en la urbanización San Isidro. Concluyendo que el alcohol recomendado es el metanol y el catalizador es el hidróxido de sodio, siendo empleados en la planta propuesta. Asimismo, no existen normas para el aceite residual de cocina, solo de aceites, que no contempla parámetros de otros países.

Palabras clave: Biocombustibles, biodiésel, aceite residual, transesterificación, valorización energética.
\end{abstract}

Abstract- The continuous pollution has generated the search for renewable energies, taking biofuels as answers, especially biodiesel from residual oil, which uses a waste that does not compete with the food sector. The objective of the research was to analyze the index of residual cooking oils for the production of biodiesel in the provinces of Lima and Trujillo. Collecting information from research works, obtaining parameters such as density, viscosity and acid number, comparing the dispersion of residual oil and biodiesel through box diagrams. Similarly, the catalysts and alcohols used were contrasted with the parameters, adding the yield. In addition, a comparison was made of the Peruvian vegetable oil regulations at an international level, as well as the research methodologies. Finally, the design of a biodiesel production plant from recycled vegetable oil in the San Isidro urbanization is proposed. Concluding that the recommended alcohol is methanol and the catalyst is sodium hydroxide, being used in the proposed plant. Likewise, there are no standards for residual cooking oil, only oils, that do not contemplate parameters from other countries.

Keywords: Biofuels, biodiesel, waste oil, transesterification, energetic valorization.

Digital Object Identifier (DOI):

http://dx.doi.org/10.18687/LEIRD2021.1.1.35

ISSN: 2414-6390 ISBN: 978-958-52071-9-6

\section{INTRODUCCIÓN}

La Agencia de Protección Ambiental de Estados Unidos muestra preocupación por la actual técnica de extracción de petróleo llamada "fracking". Asegura que puede generar estrés hídrico, contaminación de las fuentes subterráneas de aguas y de la superficie resultantes de derrames. Incluso ocasiona una contaminación atmosférica resultante de la liberación de compuestos orgánicos volátiles, y gases de efecto invernadero [1]. El instrumento legal que obliga a los países desarrollados a cumplir con los objetivos de reducción de al menos un $5.0 \%$ de estos gases es el protocolo de Kioto. El cual estuvo en vigencia desde 2008 hasta 2020 [2].

Pese a los esfuerzos no se ha logrado detener el aumento de la contaminación a la atmósfera [3] obligando a firmar nuevos acuerdos como el de París, he incluso en Perú se creó la Ley Marco sobre Cambio Climático [4]. Asimismo, el precio oscilante del petróleo a nivel internacional y los significativos impactos en las economías de los países en vías de desarrollo, la búsqueda de alternativas con la capacidad de disminuir la dependencia del carburante es un requerimiento urgente [5]. O caso contrario, pueda fusionarse con el diésel para disminuir poco a poco su uso.

La Organización de las Naciones Unidas para la Educación, la Ciencia y la Cultura afirma que el biodiésel obtenido a partir de grasas animales y aceite de cocina usado, puede reducir esencialmente las emisiones de gases de efecto invernadero a diferencia de la gasolina y el diésel mineral. Caso contrario es el biodiésel que se obtiene con fuentes que compitan con el sector alimenticio ya que, esta entidad en 2017 también afirma que con las actuales condiciones para la de producción, se requiere un promedio de 2500 litros de agua para obtener un litro de biocombustible [6]. Asimismo, en parte de Latinoamérica, los agricultores se vieron incentivados a tener cultivos para obtener combustible en lugar de utilizarlos en comida, generando una escasez que infla el mercado y cambia el uso de la tierra de los agricultores [7]. Es entonces que surge la búsqueda de una fuente que no compita directamente con el sector alimenticio.

La cocción de alimentos se genera en pocas cantidades en domicilios [8]; pero, se vuelve un gran problema al sumar los vertimientos que se generan en una ciudad muy poblada. En la industria alimentaria y en el sector hotelero; el aceite, es un 
insumo muy utilizado [9]. Sin embargo, la OMS, recalca que, un aceite no puede reutilizarse más de tres o cuatro veces en una fritura porque se vuelve altamente cancerígeno [10]. No obstante, la alta demanda de frituras genera grandes cantidades de este residuo, que, al no poder ser reutilizado en la cocina, es desechado por el alcantarillado [11]. El Organismo de Evaluación y Fiscalización Ambiental (OEFA) en el diario El Comercio remarcó que un litro de aceite usado que se echa a la alcantarilla contamina hasta mil litros de agua [12].

La reciente actualización de la estadística de biocarburantes que elabora la Comisión Nacional de los Mercados y la Competencia (CNMC) afirma que China lidera la lista de los países de procedencia de la materia prima con la que se produce el biodiésel durante la pandemia COVID-19 que se emplea en España con un 18,7 \%. Siguiéndole Indonesia $(17.6 \%)$, Argentina (12\%), Malasia (11.6\%) y Brasil (8.2\%). De los países sudamericanos llega aceite de soja y de los asiáticos aceite de palma. La causa de su primer lugar, es que la materia prima proveniente de China son aceites residuales de cocina, la primordial que se ha utilizado en 2020 para elaborar biodiésel. Esta tendencia ha hecho que países orientales, sobre todo China ingrese de lleno en el mercado del biodiésel en Europa con estos aceites.

Inclusive, España no puede abastecerse con aceite residual porque según Manuel Bustos director de la sección de Biocarburantes de la Asociación de Empresas de Energías Renovables (APPA), el COVID-19 ha influenciado considerablemente en la disminución de la producción de aceites de cocina utilizados en España, debido a las suspensiones y limitaciones que perjudicaron a la restauración y hostelería por la pandemia, esto ha hecho buscar abastecimiento en otros países. Incluso, la primordial productora europea de biocarburantes con aceite residual, informó que se estaba retirando de la elaboración de hidrobiodiésel debido a una mínima disponibilidad de aceite residual puesto que varios restaurantes están clausurados [13].

Por otro lado, en Sudamérica gran parte de biodiésel procede del aceite de soja; este se ha duplicado desde 2015 y en 2019 representó el 6\% de todo lo elaborado en Europa. Pese a ello, la soja está directamente asociada con la deforestación en Sudamérica [14]. Muchos países en el mundo, entre ellos el Perú, obligan a que las gasolinas tengan un porcentaje de biodiésel para luchar contra la contaminación [15]. Sin embargo, el avance no se ha visto cuantificado debido a que un $80 \%$ del aceite residual va al desagüe y luego al mar. El resto es reutilizado y despachado en el mercado negro [16].

Según el Instituto Nacional de Estadística e Informática, cerca de 50000 restaurantes son formales en Lima que, adicionando los informales, generarían todos los días altos números de galones de aceite quemado. Además, el Servicio de Agua Potable y Alcantarillado de Lima informa que han sucedido 157 mil emergencias en su red de alcantarillado. Las consecuencias de que las grasas sean desechadas a las redes de drenaje, es que se aglomeran en la parte interna de la tubería, disminuyendo el diámetro y grosor de las tuberías. Del mismo modo, los residuos y objetos sólidos se atrancan con la grasa acumulada, generando los atoros y después los aniegos [17].
La Ley Orgánica de Municipalidades establece que los gobiernos locales poseen autonomía política, económica y administrativa en los asuntos de su competencia, señalando que las municipalidades tienen la facultad de ejercer actos de gobierno administrativos y de administración. Esta entidad puede declarar la obligatoriedad de contar con trampas de grasa en los establecimientos comerciales del distrito [18]. Algunos ejemplos son la Municipalidad Distrital de Surquillo, De Chiclayo, La Esperanza, etc. Sin embargo, va a depender de la municipalidad si requiere la implementación de esta, puesto que, no todos los distritos cuentan con ordenanzas.

Esta realidad demuestra que la contaminación de aceites residuales se convierte en un problema severo en Perú; sobre todo en la capital, dado que este insumo es vertido descontroladamente, es entonces que al convertir estos en una materia prima para la producción de biodiésel, entran en los objetivos de desarrollo sostenible de la ONU, en la categoría de energía asequible y no contaminante, así como en acción por el clima [19]. Por esas cualidades, es importante ahondar en investigaciones enfocadas desde diferentes puntos de vista que demuestren la importancia de reutilizar el aceite de cocina y la producción de biodiésel para evitar la contaminación.

\section{MetodoloGíA}

El enfoque considerado para esta investigación es cuantitativo, ya que muestran resultados de los diferentes parámetros fisicoquímicos (índice de acidez, densidad, viscosidad y rendimiento) de las investigaciones, comparando las distribuciones de los datos. Asimismo, es descriptiva debido a que describe la realidad del aceite peruano que es empleado muchas veces en procesos de cocción y luego desechado por el alcantarillado.

Con respecto a la muestra, este estudio usó una revisión de 30 artículos, quedando 15 investigaciones sobre índices de aceites residuales de cocina para la producción de biodiésel en las provincias de Lima y Trujillo debido a la aplicación de diferentes tipos de criterios de selección, empezando por buscar con las variables de aceite residual proveniente de cocinas y sobre el biodiésel, en diferentes repositorios de universidades.

Se creó una base de datos de investigaciones, cuyos datos se basaron en transesterificación, permitiendo describir la comparación entre las características del aceite residual como del biodiesel. Con esta información se obtuvo el índice de aceite residual de cocina que se pueden utilizar en la producción de biodiésel; incluso, se aprovechó el software estadístico para representar la información mediante figuras y tablas, concretando así el primer objetivo. Asimismo, la data presente también sirvió para comparar los catalizadores y alcoholes, observando su influencia en los parámetros fisicoquímicos.

Por otra parte, se realizó una comparación de la normativa del aceite vegetal a nivel nacional e internacional. También, se compararon las metodologías de las diferentes investigaciones en la producción de biodiésel a partir de aceites residuales de cocina en las provincias de Lima y Trujillo.

Por último, se propone el diseño de una planta de producción de biodiésel a partir de aceite vegetal reciclado en la urbanización San Isidro. Para evaluar la propuesta se 
utilizaron los datos procedentes de diseños de planta de biodiesel modelados a través del software SketchUp en su versión libre. Asimismo, se considera un sobredimensionamiento de la planta, debido a que la pandemia COVID - 19 no permite el trabajo al $100 \%$ de los restaurantes en la zona de estudio.

\section{RESULTADOS Y DISCUSIÓN}

La investigación se basa en la reutilización del aceite residual de cocina para la producción de biodiésel, y así emplearse como un biocombustible que reduce el grado de contaminación que dicho residuo genera. Así también, lo asevera la teoría de la economía circular que permite la reutilización de los productos disminuyendo los residuos [20], la valorización energética al ser utiliza un desecho como combustible [21] y el desarrollo sostenible que satisface las necesidades de la generación presente sin comprometer los recursos de las futuras generaciones [22].

Con el objetivo de analizar el índice de aceites residuales de cocina para la producción de biodiésel se analizaron los parámetros de índice de acidez, densidad y viscosidad de los resultados de las diferentes investigaciones. La figura 1, muestra un mayor rango para el aceite residual que en el biodiésel. El cual se encuentra fuera de los valores que brinda la Norma Técnica Peruana 209.001:1983 Aceites y vegetales comestibles, por no tener resultados inferiores a $0.2 \mathrm{mg} \mathrm{KOH} / \mathrm{g}$.

Del mismo modo, la Norma Técnica Peruana 321.125:2008 Especificaciones Técnicas Biodiésel indica que deben ser menor a $0.5 \mathrm{mg} \mathrm{KOH} / \mathrm{g}$, de los cuales de los diferentes trabajos solo dos resultados cumplen. Por tal motivo la realidad es que el aceite ha sido sometido cocción intensa por periodos de tiempo más largos de lo usual, de manera similar a artículos de México donde todas las muestras de los diferentes tipos de restaurantes superaron el rango establecido por el mismo motivo [23].

De forma similar, el aceite residual de los puestos de comida del mercado Municipal de Huamachuco sobrepasa el límite oscilando en un rango desde $1.965 \mathrm{mg} \mathrm{KOH} / \mathrm{g}$ hasta $2.923 \mathrm{mg}$ $\mathrm{KOH} / \mathrm{g}$. Esto indica que los aceites no son comestibles y causan un daño a la salud. Mientras que un alto índice de acidez del biodiésel se puede deber a que el aceite se empleó sin ningún tratamiento previo [24]; por otra parte, un alto índice de acidez en el biodiésel indica que el biocombustible es corrosivo y puede generar daños en el motor [25].

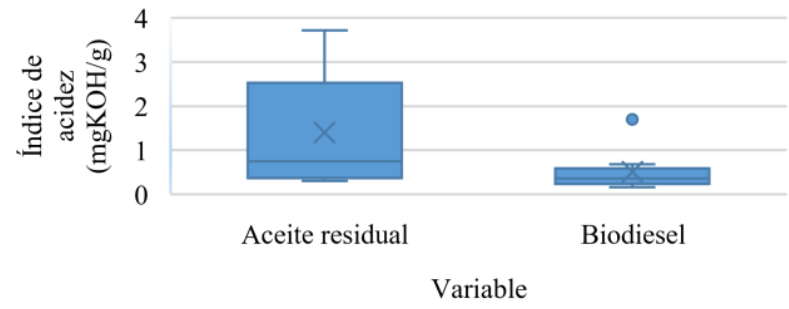

Fig. 1 Diagrama de cajas y bigotes del índice de acidez de los aceites residuales de cocina y del biodiésel obtenido en las provincias de Lima y Trujillo
La figura 2 presenta un rango con más distribución en la densidad para el aceite residual con un dato atípico con 0.816 $\mathrm{g} / \mathrm{ml}$, a diferencia del biodiésel con dos valores atípicos, tanto de manera superior $\left(\mathrm{N}^{\circ} 29\right) 0.975 \mathrm{~g} / \mathrm{ml} \mathrm{e}$ inferior de $0.85 \mathrm{~g} / \mathrm{ml}$. Es uno de los parámetros que se miden usualmente, confirmándose en la investigación de Herrera que obtuvo una densidad inicial de $0.916 \mathrm{~g} / \mathrm{ml}$ de aceite residual y $0.876 \mathrm{~g} / \mathrm{ml}$ de biodiésel [26].

Si bien no existe límite en nuestra normativa peruana para este parámetro, la norma europea establece un rango entre 0.86 y $0.9 \mathrm{~g} / \mathrm{ml}$ para el biodiésel y su importancia es vital debido a que un valor bajo produce un aumento del consumo del carburante, teniendo solo 1 resultado $(0.85 \mathrm{~g} / \mathrm{ml})$ que no cumplen lo recomendado. Por otra parte debido a que la densidad del aceite es inferior a la del agua, al ser desechados, se generan capas en la superficie de los reactores biológicos, perjudicando al sistema [27].

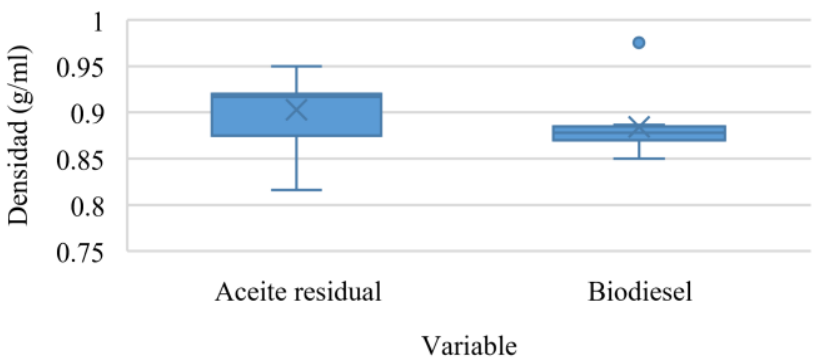

Fig. 2 Diagrama de cajas y bigotes de la densidad de los aceites residuales de cocina y del biodiésel obtenido en las provincias de Lima y Trujillo

La normativa peruana contempla la viscosidad en un rango de 1.9 a $6 \mathrm{~mm}^{2} / \mathrm{s}$, siendo 4 los resultados fuera del límite. A nivel europeo debe encontrarse entre 3.5 a $5 \mathrm{~mm}^{2} / \mathrm{s}$, teniendo 6 valores que sobrepasarían el rango. La importancia de la viscosidad se debe a que, si es elevada en el biodiésel, la combustión es incompleta; a diferencia de una baja, que puede producir fugas del combustible, reduciendo la eficiencia [25].

La distribución de este parámetro puede ser observado en la figura 3, donde al igual que en los anteriores casos aceite residual tiene un rango más amplio que el biodiésel. Los valores altos del aceite residual se deben a las reacciones termolíticas y oxidativas que suceden durante el proceso de freído que pueden afectar la producción en el contacto entre las moléculas de aceite y metanol, provocando baja conversión de los triglicéridos [28].

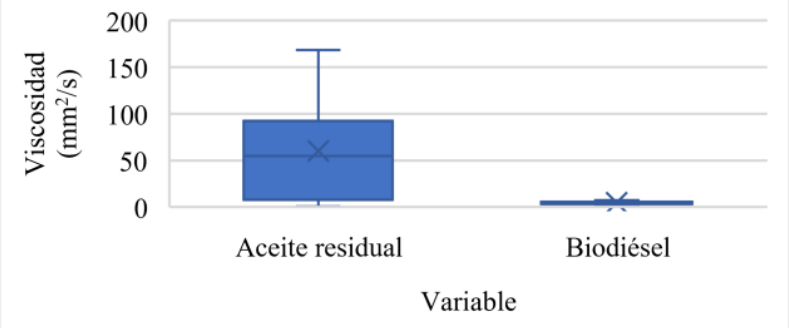

Fig. 3 Diagrama de cajas y bigotes de la viscosidad de los aceites residuales de cocina y del biodiésel obtenido en las provincias de Lima y Trujillo 
Por otra parte, se logró contrastar los alcoholes empleados en la producción de biodiésel a partir de aceite residual de cocina en las provincias de Lima y Trujillo, siendo el metanol y el etanol los únicos utilizados. En esta ocasión, las gráficas de cajas fueron para dos variables que, a la vez, se compraban con los parámetros fisicoquímicos de índice de acidez, densidad, viscosidad y rendimiento representados por las figuras 4, 5, 6 y 7 respectivamente.

En la comparación de los alcoholes en el índice de acidez se observa un rango amplio para el metanol a diferencia del etanol. La figura 4 muestra que a lo largo de las investigaciones el metanol abarca un mayor rango y valores más bajos que el etanol, debido a que el aumento del $\mathrm{pKa}$ supone una disminución de la acidez [29]. Esto tiene un gran impacto al elegir entre ambos alcoholes ya que este parámetro tiene que ser menor a $0.5 \mathrm{mgKOH} / \mathrm{g}$, siendo cumplido a través del metanol en la mayoría de casos.

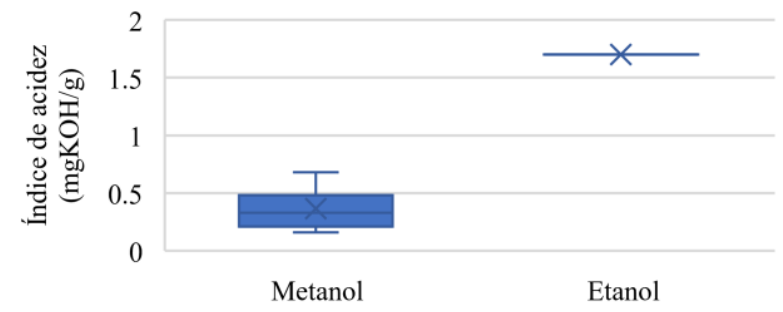

Alcohol

Fig. 4 Diagrama de cajas y bigotes del índice de acidez del biodiésel comparando los alcoholes empleados en las provincias de Lima y Trujillo

En cuanto a la densidad la figura 5 indica que el etanol presenta un rango más amplio y valores menores a diferencia del metanol. Esta diferencia mínima se debe a que el etanol es menos denso, siendo reflejado en la densidad del biodiésel que se obtiene. La densidad del metanol es de $0.792 \mathrm{~g} / \mathrm{ml}$ mientras que el del etanol es de $0.789 \mathrm{~g} / \mathrm{ml}$ [30]. Si se requiere una mayor densidad en el biodiésel, el metanol sería el alcohol idóneo; a diferencia que, si se necesita una menor densidad, el etanol debería ser el seleccionado.

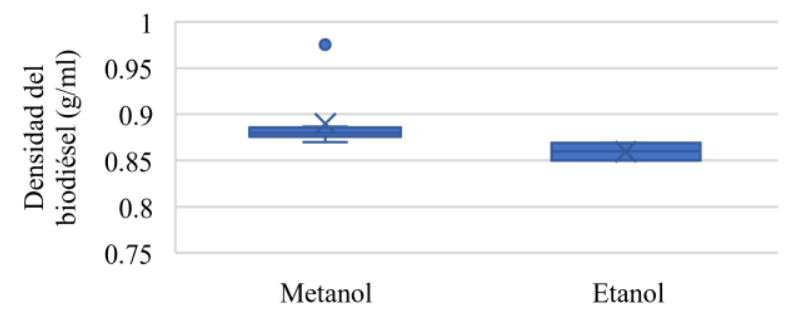

Alcohol

Fig. 5 Diagrama de cajas y bigotes de la densidad del biodiésel comparando los alcoholes empleados en las provincias de Lima y Trujillo

Asimismo, la influencia de la viscosidad de los alcoholes se ve reflejada en la figura 6 , el etanol posee una viscosidad que es mucho mayor que la del metanol. El primero posee un valor de $1.52 \mathrm{~mm}^{2} / \mathrm{s}$ aumentando así la viscosidad del biodiésel, caso contrario al segundo, que posee una viscosidad $0.742 \mathrm{~mm}^{2} / \mathrm{s}$ [31].

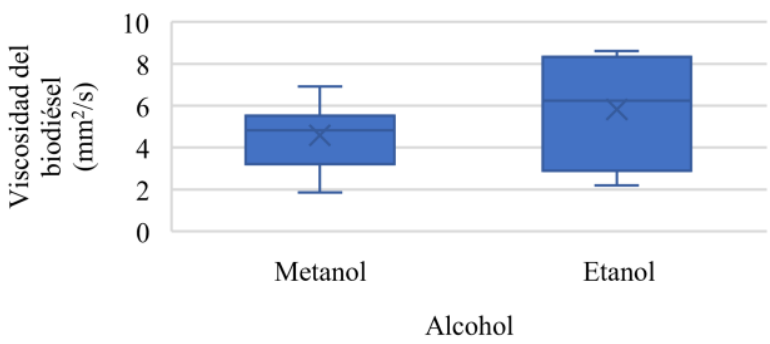

Fig. 6 Diagrama de cajas y bigotes de la viscosidad del biodiésel comparando los alcoholes empleados en las provincias de Lima y Trujillo

A diferencia del rendimiento en la figura 7 donde el metanol presenta valores más altos que el etanol. Corroborando en tesis donde el rendimiento con metanol es mayor (97\%) al de etanol (95\%) [32]. Concluyendo que el proceso con mayor rendimiento sea aceite residual o nuevo es el metanol. Debido a que la preparación de biodiésel es más sencilla con este insumo; sin embargo, es más restringido por ser altamente tóxico. Además, la obtención del biocombustible con etanol no ha sido muy estudiado, siendo reflejado a través de los resultados donde la mayoría utiliza metanol que etanol.

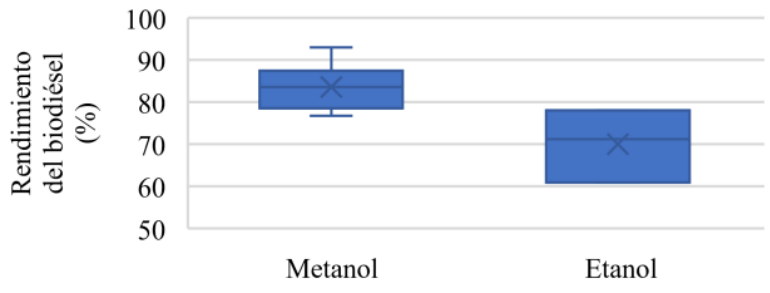

Alcohol

Fig. 7 Diagrama de cajas y bigotes del rendimiento del biodiésel comparando los alcoholes empleados en las provincias de Lima y Trujillo

Al contratar los catalizadores en la producción del biocombustible, se observa en la figura 8 que en el índice de acidez el biodiésel elaborado con hidróxido de potasio $(\mathrm{KOH})$ tiene más rango. Así como valores que sobrepasan lo establecido en la Norma Técnica Peruana para biodiésel, a diferencia del hidróxido de sodio donde los resultados se encuentran dentro del límite establecido.

En el índice de acidez se utiliza hidróxido de potasio para neutralizar ácidos grasos presentes en un gramo de aceite [33]. Esto se ve reflejado al utilizar el $\mathrm{KOH}$ como catalizador, llegando a un rango más amplio que al utilizar $\mathrm{NaOH}$ 


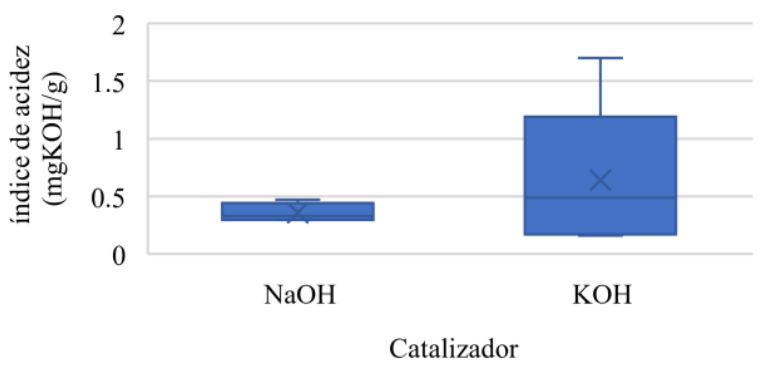

Fig. 8 Diagrama de cajas y bigotes del índice de acidez del biodiésel comparando los catalizadores empleados en las provincias de Lima y Trujillo

En la figura 9 se aprecia una mayor dispersión para la densidad por parte del $\mathrm{KOH}$. Las densidades de ambos catalizadores son similares, sin embargo, al utilizarlos para elaborar el biodiésel, el que emplea KOH llega a una densidad más baja. Las densidades de ambos catalizadores presentan una leve diferencia, siendo el del $\mathrm{NaOH} 2.13 \mathrm{~g} / \mathrm{ml}$ y del $\mathrm{KOH} 2.12$ $\mathrm{g} / \mathrm{ml}$ [34]. Logrando así, al emplear hidróxido de potasio, densidades más bajas, pero no significativas.

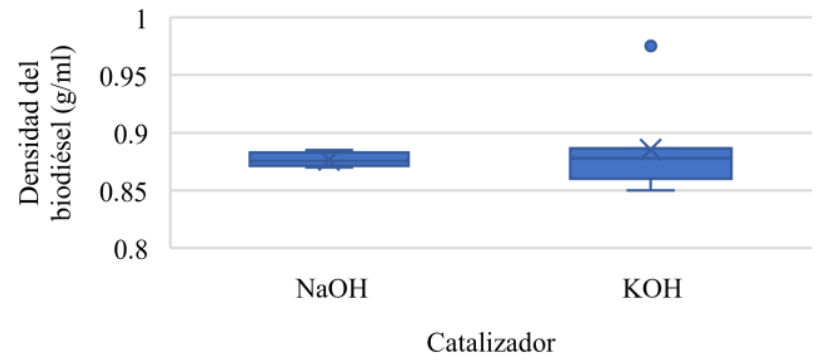

Fig. 9 Diagrama de cajas y bigotes de la densidad del biodiésel comparando los catalizadores empleados en las provincias de Lima y Trujillo

Mientras que en la figura 10, en la viscosidad las dispersiones se encuentran casi igualadas, teniendo el $\mathrm{NaOH}$ como catalizador que puede llegar a valores más bajos. Este parámetro, va a tener una mayor dependencia del aceite que del catalizador; sin embargo, es preferible tener una viscosidad baja que alta, por los daños al motor se requiere una viscosidad mínima para evitar pérdidas durante la inyección del combustible ya que un combustible muy viscoso puede causar una mala atomización, que lleva a mala combustión y formación de depósitos [35].

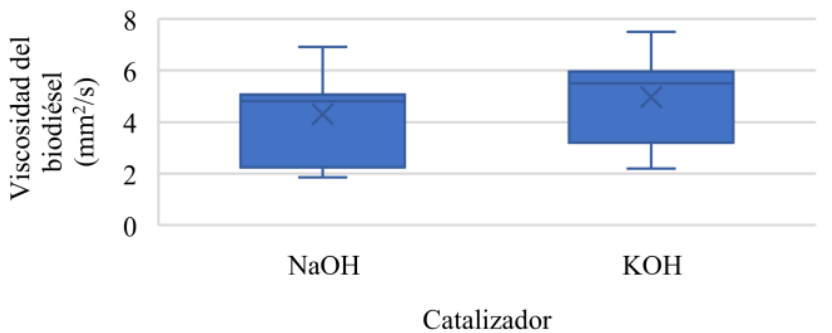

Fig. 10 Diagrama de cajas y bigotes de la viscosidad del biodiésel comparando los catalizadores empleados en las provincias de Lima y Trujillo
En la figura 11 resalta el $\mathrm{NaOH}$ en el rendimiento donde obtiene valores más altos en su dispersión. Siendo confirmado por investigaciones, ya que el empleo de $\mathrm{KOH}$ genera mayor existencia de jabones (reacción de saponificación) y, por ende, se muestra un menor rendimiento al contrastarlo con el hidróxido de sodio [36].

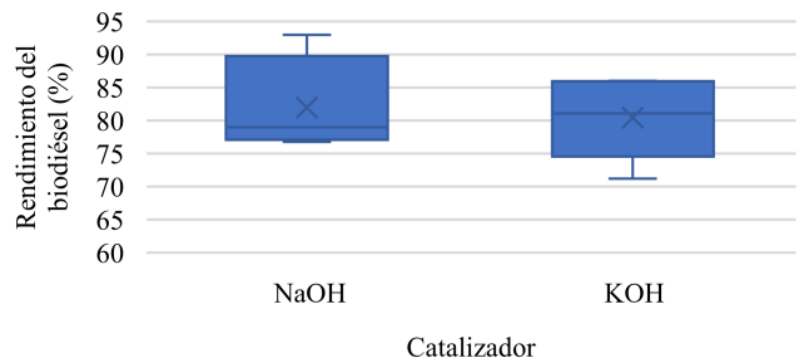

Fig. 11 Diagrama de cajas y bigotes del rendimiento del biodiésel comparando los catalizadores empleados en las provincias de Lima y Trujillo

En la tabla $\mathrm{N}^{\mathrm{o}} 1$, al realizar una comparación de la Normativa Peruana de Aceite vegetal a Nivel Sudamericano, se observa que la normativa del aceite vegetal nacional no contiene varios parámetros, a diferencia de otros países. En Perú, la NTP 209.001.1983 contempla la humedad y materia volátil $\left(105^{\circ} \mathrm{C}\right)$ con un valor de $0.1 \%$, sin embargo, esté límite es más estricto en Venezuela (CONVENIN 30:1997) con 0.05 $\%$ y en Colombia (Resolución Ministerial 0002154) es más permisible con 0.2, siendo Ecuador (NTE INEN 34:2012) el país que no presenta un límite. La importancia de su determinación es por cuestiones de calidad y salud pública, puesto que es la base de referencia que permite comparar características nutrimentales en alimento [37].

Otro parámetro que solo contemplan Venezuela y Colombia, son las impurezas insolubles, definidas como cantidad de suciedad y otras materias extrañas obtenidas [38], para Colombia el límite es $0.05 \%$, igual que para Venezuela. Asimismo, el contenido de jabón para el país colombiano debe ser negativo, mientras que para los venezolanos es de $3 \mathrm{mg} / \mathrm{kg}$. Perú y Ecuador no presentan información sobre estos parámetros

Un parámetro que todos los países presentan es el índice de peróxido (meq O2/ Kg) para Perú, Venezuela y Colombia debe ser de 5, siendo más permisible Ecuador con 10. Este es una medida del estado de oxidación de un aceite o grasa; y aumenta después del proceso de freído. Los peróxidos derivados de los lípidos durante la fritura se integran en parte dentro del alimento con efectos perjudiciales para el consumidor, tales como cambios celulares irreversibles, mutación, cáncer y aterosclerosis,

De igual manera, la acidez también es considerada en todas las normativas, teniendo un valor de 0.1 en Colombia y Venezuela, mientras que en Perú y Ecuador debe ser menor a 0.2. El aumento de esta sucede cuando el aceite de fritura no se renueva habitualmente, esto origina la generación de humos y sabores indeseables, y ocasiona la rancidez hidrolítica e incluso enfermedades [39]. 
TABLA I

Comparación de la Normativa Peruana de Aceite vegetal a Nivel SUDAMERICANO

\begin{tabular}{|c|c|c|c|c|}
\hline Parámetros & Colombia & Ecuador & Perú & Venezuela \\
\hline $\begin{array}{c}\text { Humedad y materia } \\
\text { volátil (\%) }\end{array}$ & 0.2 & - & 0.1 & 0.05 \\
\hline $\begin{array}{c}\text { Impurezas insolubles } \\
(\%)\end{array}$ & 0.05 & - & - & 0.05 \\
\hline $\begin{array}{c}\text { Contenido de jabón } \\
(\mathrm{mg} / \mathrm{kg})\end{array}$ & Negativo & - & - & 3 \\
\hline $\begin{array}{c}\text { Índice de peróxido } \\
(\text { meq O2/ Kg) }\end{array}$ & 5 & 10 & 5 & 5 \\
\hline Acidez (\%) & 0.1 & $<0.2$ & $<0.2$ & 0.1 \\
\hline $\begin{array}{c}\text { Índice de refracción } \\
\left(25^{\circ} \mathrm{C}\right)\end{array}$ & - & 1.476 & - & $1.463-$ \\
\hline $\begin{array}{c}\text { Pérdida por } \\
\text { calentamiento (\%) }\end{array}$ & - & 0.05 & - & - \\
\hline $\begin{array}{c}\text { Densidad Relativa } \\
\left(20^{\circ} \mathrm{C}\right)\end{array}$ & - & - & - & $0.8969-$ \\
\hline \multicolumn{2}{|c|}{} & & & 0.926 \\
\hline
\end{tabular}

En tal sentido, después de la comparación de las normativas se puede observar un vacío en varios parámetros, ya que no son consideraros dentro de la legislación peruana, contemplando solo la Humedad y materia volátil, el índice de peróxido y la acidez. Parámetros no suficientes en otros países, debido a que las impurezas insolubles, contenido de jabón, índice de refracción, pérdida por calentamiento y densidad aseguran una mayor calidad en el producto y evitan enfermedades que se puedan causar posteriormente.

Perú no cuenta con una norma para considerar rancio un aceite, es más ninguno de los países mencionados tiene una normativa para considerar a un aceite como residual. Es por eso que se comparan los resultados con los límites establecidos por las normas técnicas.

La tabla $\mathrm{N}^{\circ} 2$ demuestra la comparación de las metodologías de las diferentes investigaciones en la producción de biodiésel a partir de aceites residuales de cocina en las provincias de Lima y Trujillo. En esta tabla se considera la cantidad de aceite que se utilizó, los catalizadores y alcoholes empleados, así como la temperatura en el proceso de transesterificación.

La mayoría de investigaciones mostraron el rendimiento al que pudo llegar el biodiésel bajo estas especificaciones. Se puede observar que el alcohol idóneo es el metanol, junto al $\mathrm{NaOH}$ como catalizador, las temperaturas en la transesterificación $\left(60^{\circ} \mathrm{C}-65^{\circ} \mathrm{C}\right)$ son las más empleadas, la metodología más eficiente fue la que alcanzó el mejor resultado que es el primero con un $93.06 \%$ de rendimiento de biodiésel, este realizó el método de transesterificación en metanol subcrítico (altas temperaturas). Otro resultado que alcanzó un rendimiento similar fue el que alcanzó un $93 \%$ de rendimiento, hidróxido de sodio y metanol, empleando una temperatura de $65^{\circ} \mathrm{C}$ en la transesterificación, de manera similar al tercer resultado que utilizó el mismo catalizador y alcohol, pero a menor temperatura, alcanzando un rendimiento de $88 \%$. En base a que estos resultados son eficientes con estos insumos, se deciden utilizar tanto el metanol como hidróxido de sodio en la presente propuesta.
TABLA II

COMPARACIÓN DE LAS METODOLOGÍAS EN LA PRODUCCIÓN DE BIODIÉSEL

\begin{tabular}{|c|c|c|c|c|c|}
\hline $\begin{array}{c}\mathrm{N}^{\circ} \\
\text { Artíc } \\
\text { ulo } \\
\end{array}$ & Aceite & $\begin{array}{c}\text { Catalizad } \\
\text { or }\end{array}$ & Alcohol & $\begin{array}{c}\text { Temperat } \\
\text { ura }\end{array}$ & $\begin{array}{c}\text { Rendimie } \\
\text { nto }\end{array}$ \\
\hline$[40]$ & $600 \mathrm{ml}$ & $\begin{array}{c}1 \% \\
\mathrm{NaOH}\end{array}$ & $\begin{array}{l}\text { Relación 9:1 de } \\
\text { metanol: aceite }\end{array}$ & $180^{\circ} \mathrm{C}$ & $93.06 \%$ \\
\hline$[41]$ & $100 \mathrm{~g}$ & $\begin{array}{c}0.5 \% \mathrm{de} \\
\mathrm{NaOH}\end{array}$ & $\begin{array}{l}\text { Relación } 6: 1 \text { de } \\
\text { metanol: aceite }\end{array}$ & $65^{\circ} \mathrm{C}$ & $93 \%$ \\
\hline$[42]$ & $100 \mathrm{ml}$ & $\begin{array}{l}0.68 \mathrm{~g} \\
\mathrm{NaOH} \\
\end{array}$ & $15 \mathrm{ml}$ metanol & $50^{\circ} \mathrm{C}$ & $88 \%$ \\
\hline [43] & $200 \mathrm{ml}$ & $\begin{array}{l}1 \mathrm{~g} \mathrm{de} \\
\mathrm{KOH}\end{array}$ & $\begin{array}{l}40 \mathrm{ml} \mathrm{de} \\
\text { metanol }\end{array}$ & $60^{\circ} \mathrm{C}$ & $87 \%$ \\
\hline$[44]$ & $41.1 \mathrm{~L}$ & $\begin{array}{l}148 \mathrm{~g} \\
\mathrm{KOH}\end{array}$ & $\begin{array}{c}12.3 \mathrm{~L} \mathrm{de} \\
\text { metanol }\end{array}$ & $60^{\circ} \mathrm{C}$ & $85.97 \%$ \\
\hline$[45]$ & $200 \mathrm{ml}$ & $\begin{array}{l}0.85 \mathrm{~g} \\
\mathrm{NaOH} \\
\end{array}$ & $40 \mathrm{ml}$ metanol & $60^{\circ} \mathrm{C}$ & $81.12 \%$ \\
\hline$[46]$ & $4 \mathrm{~L}$ & $\begin{array}{c}28 \mathrm{~g} \\
\mathrm{NaOH} \\
\end{array}$ & $\begin{array}{c}800 \mathrm{ml} \mathrm{de} \\
\text { metanol }\end{array}$ & $60^{\circ} \mathrm{C}$ & $80 \%$ \\
\hline$[47]$ & $150 \mathrm{ml}$ & $\begin{array}{c}0.85 \mathrm{~g} \mathrm{de} \\
\mathrm{KOH}\end{array}$ & $\begin{array}{c}100 \mathrm{ml} \mathrm{de} \\
\text { etanol }\end{array}$ & $70^{\circ} \mathrm{C}$ & $78 \%$ \\
\hline [48] & $200 \mathrm{~g}$ & $\begin{array}{l}0.15 \mathrm{~g} \\
\mathrm{KOH}\end{array}$ & $\begin{array}{c}1.09 \mathrm{ml} \mathrm{de} \\
\text { metanol }\end{array}$ & $50^{\circ} \mathrm{C}$ & $76.80 \%$ \\
\hline [49] & $15 \mathrm{~g}$ & $\begin{array}{c}2.5 \mathrm{~g} \mathrm{de} \\
\mathrm{KOH}\end{array}$ & $60 \mathrm{ml}$ de etanol & $60^{\circ} \mathrm{C}$ & $71.20 \%$ \\
\hline [50] & $200 \mathrm{ml}$ & $\begin{array}{c}6 \% \mathrm{de} \\
\mathrm{CaO}\end{array}$ & $\begin{array}{l}\text { 15:1 de relación } \\
\text { etanol: aceite }\end{array}$ & $70^{\circ} \mathrm{C}$ & $60.90 \%$ \\
\hline [51] & $40 \mathrm{ml}$ & $\begin{array}{c}0.9 \mathrm{~g} \\
\mathrm{NaOH}\end{array}$ & $11 \mathrm{ml}$ metanol & $\begin{array}{c}65^{\circ} \mathrm{C}-70 \\
{ }^{\circ} \mathrm{C}\end{array}$ & - \\
\hline$[52]$ & $2 \mathrm{~L}$ & $\begin{array}{l}28 \mathrm{~g} \\
\mathrm{KOH}\end{array}$ & $\begin{array}{c}800 \mathrm{ml} \mathrm{de} \\
\text { etanol }\end{array}$ & $60^{\circ} \mathrm{C}$ & - \\
\hline$[53]$ & $1.5 \mathrm{~L}$ & $\begin{array}{l}6.70 \mathrm{~g} \\
\mathrm{KOH}\end{array}$ & $\begin{array}{c}568 \mathrm{ml} \mathrm{de} \\
\text { etanol }\end{array}$ & $65^{\circ} \mathrm{C}$ & - \\
\hline
\end{tabular}

Para finalizar, se describe la propuesta del diseño de una planta de biodiésel en la urbanización San Isidro, Trujillo La ejecución de esta propuesta es vital para evitar el atascamiento de las redes de alcantarillado, disminuir el impacto ambiental negativo en los ecosistemas acuáticos; principalmente en las fuentes hídricas nacionales, así como disminuir la reutilización de los aceites al cocinar ya que son nocivos para la salud humana y en lugar de eso utilizarlos en la producción de biodiésel. Por tal motivo, se considera recolectar el aceite residual que los restaurantes de este distrito generan luego de los periodos de cocción. Teniendo un punto de acopio para a recolección del aceite, brindando a los centros de comida la oportunidad de convertirse en ecoamigables.

La metodología está basada en el diseño y construcción de una planta de producción de Biodiésel a partir de aceite vegetal reciclado [54], así como también se emplea una selección de investigaciones con metodologías eficientes, escogiendo al metanol como alcohol y el hidróxido de sodio como catalizador, de tal manera que se pueda obtener biodiésel de un buen rendimiento con parámetros dentro de los límites.

La capacidad de aceite que puede tratar la planta es de 80 litros semanal, cantidad que sobrepasa a lo producido por los restaurantes (40 litros), esto es debido a la realidad a la cual nos enfrentemos (COVID - 19), pues una vez retomado el nivel de consumo a su totalidad se espera una mayor generación de aceite residual, ayudando la planta a reducir el nivel de contaminación en el que se encuentra la ciudad de Trujillo. 
Para llegar al diseño de la planta de producción de biodiesel, se tuvo que delimitar el área de estudio mediante un mapa para identificar los restaurantes participantes, establecer especificaciones técnicas básicas para el funcionamiento de la planta de biodiésel y realizar un modelado geométrico de la planta en tercera dimensión.

El plan de recojo del aceite será realizado por un operario que concurrirá a los distintos puntos de recojo en la urbanización San Isidro. La materia prima esencial del proceso es el aceite, para ello es de vital importancia tener una cantidad de suministradores que sean estables, que abastezcan a la planta de producción de biodiésel con los litros de aceite necesarios para una generación de biocombustible continua.

La planta estará localizada en la urbanización San Isidro ubicado en el distrito de Trujillo, provincia de Trujillo, departamento La libertad, en Perú Dentro de la urbanización en un área de $0.38 \mathrm{~km}^{2}$, existen alrededor de 15 pequeños restaurantes, representados en la siguiente figura:

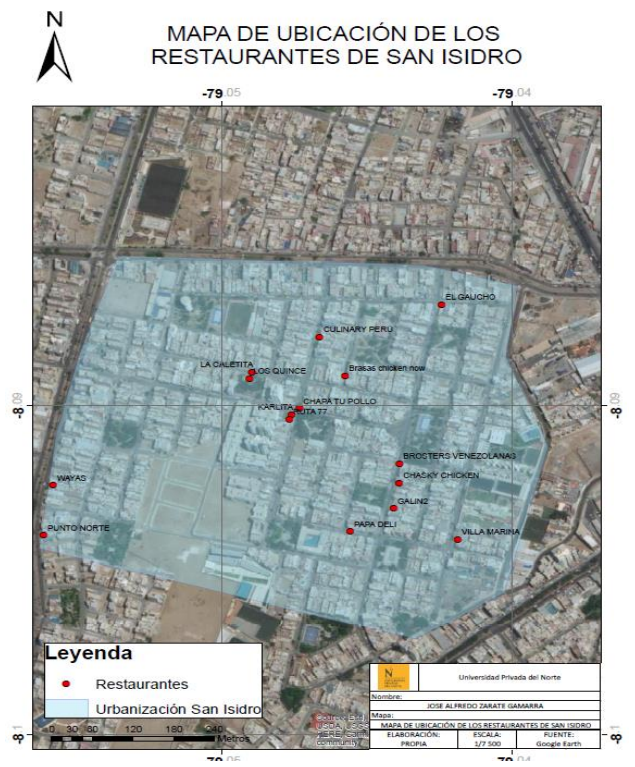

Fig. 12. Mapa de ubicación de los restaurantes de San Isidro

Los insumos necesarios son escogidos gracias a la metodología de las diferentes tesis, escogiendo al hidróxido de sodio como catalizador, el metanol como alcohol y los aceites vegetales usados. Además, se necesita un suministro continuo de agua y energía. Los equipos necesarios son el tanque de almacenamiento de aceite (T1), el tanque de químicos (T2), el tanque de procesamiento (T3), bombas, motores, tuberías y un tablero eléctrico.

El dimensionamiento ocupa un espacio físico de 2 metros 40 centímetros de largo, 80 centímetros de ancho y 1 metro 80 centímetros de alto. Teniendo como Emplazamiento total de la planta requiere de un área de aproximadamente $12.6 \mathrm{~m}^{2}$. Además, en caso de una mayor generación de aceite residual que exceda la planta, se considera espacio suficiente para conectar una mayor cantidad de tanques que permitan el confrontar un posible repunte de alta demanda en el rubro restaurantero.

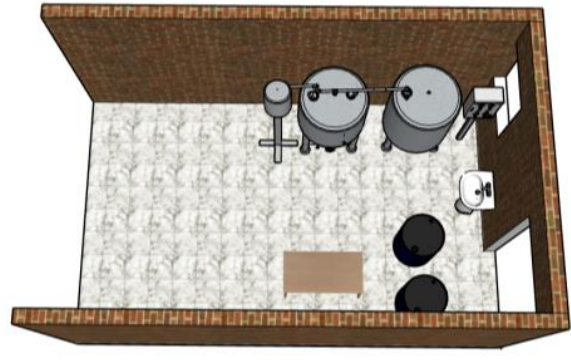

Fig. 13 Modelado geométrico de la planta de biodiésel en tercera dimensión

El almacenamiento y precalentado del aceite se realiza en el tanque 1, la filtración del aceite es llevada a cabo a través de una malla de $80 \mu \mathrm{m}$ y una tela. El precalentamiento del aceite tiene como objetivo reducir la viscosidad para favorecer el transporte del líquido antes del procesamiento. En vista de que es un proceso netamente de precalentado, se debe mantener una temperatura que se aproxime a $\operatorname{los} 30^{\circ} \mathrm{C}$.

En el tanque 3, la fase de secado comprende el aumento de temperatura del tanque en el cual se encuentra el aceite purificado y precalentado con una temperatura cercana a los $95^{\circ} \mathrm{C}$. Después del proceso es indispensable que la temperatura del aceite se reduzca hasta $60^{\circ} \mathrm{C}$, siendo esta la temperatura a la que más adelante se efectúa el método de transesterificación.

El proceso de titulación del aceite, tiene como objetivo obtener el grado de acidez presente en el aceite, a través de este determinar la cantidad de hidróxido de sodio que se tiene que emplear en la transesterificación [55].

En el momento en que la temperatura logre alcanzar los $60^{\circ} \mathrm{C}$ se comienza a agregar la mezcla del catalizador y alcohol del tanque de químicos (T2) al tanque de procesamiento (T3). Es preciso encender los motores durante la entrada de la mezcla química al tanque que se regulariza a través de controladores de velocidad. Se tiene que efectuar la mezcla a lo largo de una duración de alrededor de 2 horas, a causa de la geometría del tanque.

Después de haber culminado la mezcla se requiere que el tanque permanezca en reposo hasta lograr la definición del proceso de transesterificación. En esta fase se obtienen dos productos, tanto ésteres (biodiésel) en la parte superior, como glicerol en la parte inferior, debido a la precipitación [56].

Al empezar el drenaje del tanque, se aprecia la precipitación de la glicerina con un color café lóbrego y viscoso, que a continuación, permitirá dar cabida al biodiésel. Una vez culminado el proceso, es obligatorio el reposo del tanque para que las partículas restantes de glicerina que no pudieron ser drenadas se logren sedimentar. El tiempo que se requiere es alrededor de 12 horas de reposo. La obtención de esta etapa se define como biodiésel crudo y aún precisa de ser lavado y purificado.

Considerando la estructura del diseño de la planta, se emplea el lavado con agua. No obstante, los riesgos de emulsión y saponificación deben ser disminuidos, por lo tanto, el lavado se realizará usando un nebulizador. El biocombustible resulta turbio y muestra burbujas, por lo que se deduce la aparición de agua. El secado del biodiésel, involucra que las moléculas de 
agua que están en suspensión en la muestra logren alcanzar la superficie a través de la evaporación, la existencia de agua en la muestra, disminuye el rendimiento del biodiésel [57].

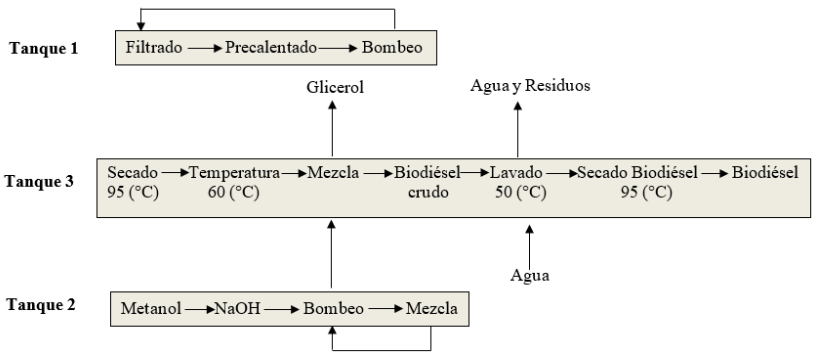

Fig. 14 Diagrama de proceso en los tanques

La cantidad máxima de aceite residual proveniente de los restaurantes de la urbanización San Isidro que se espera recolectar a la semana es de 40 litros, llegando a un número mensual de 160 litros. La cantidad anual de aceite reciclado puede alcanzar los 640 litros, ayudando a preservar el ambiente. El subproducto que se obtiene de la producción del biodiésel es la glicerina, teniendo varios fines de uso como jabón, en biogás y combustible, e incluso como estiércol [54].

\section{CONCLUSIONES}

El análisis del índice de aceites residuales de cocina para la producción de biodiésel en las provincias de Lima y Trujillo muestra una mayor distribución de dispersión de datos para el aceite residual de cocina mediante la gráfica de cajas en sus diferentes parámetros de índice de acidez $(0.3$ a $3.72 \mathrm{mg}$ $\mathrm{KOH} / \mathrm{g}$ ), densidad (0.86 a $0.95 \mathrm{~g} / \mathrm{ml}$ ) y viscosidad (0.4 a 168.3 $\left.\mathrm{mm}^{2} / \mathrm{s}\right)$.

Al contrastar los catalizadores se observa que el que tiene un mayor rango es el hidróxido de potasio $(\mathrm{KOH})$ en el índice de acidez al estar entre 0.159 y $1.9 \mathrm{mg} \mathrm{KOH} / \mathrm{g}$, en la densidad con 0.85 a $0.89 \mathrm{~g} / \mathrm{ml}$ y en la viscosidad, que abraca desde 2.2 hasta $7.5 \mathrm{~mm}^{2} / \mathrm{s}$. Sin embargo, el rendimiento es mayor en el $\mathrm{NaOH}$, que llega hasta $93.06 \%$. Mientras que, en los alcoholes, el metanol presenta un amplio rango en el índice de acidez al estar entre 0.159 y $0.68 \mathrm{mg} \mathrm{KOH} / \mathrm{g}$, la densidad con 0.87 hasta $0.89 \mathrm{~g} / \mathrm{ml}$ junto a un rendimiento más alto, abarcando desde un $76.8 \%$ hasta un $93.06 \%$. A diferencia del etanol que posee una mayor viscosidad contemplada entre 2.2 a $8.6 \mathrm{~mm}^{2} / \mathrm{s}$

La comparación de la normativa del aceite vegetal demuestra que Perú no considera parámetros que son esenciales en otros países para el control y calidad del producto, así como para evitar perjuicios a la salud.

Las investigaciones más eficientes en la producción de biodiésel a partir de aceites residuales de cocina en las provincias de Lima y Trujillo fueron las que utilizaron metanol como alcohol e hidróxido de sodio como catalizador, alcanzando un rendimiento alto, con una temperatura cercana a los $60^{\circ} \mathrm{C}$ en la transesterificación.

La propuesta de la planta de biodiésel en la urbanización San Isidro, Trujillo, está enfocada en reutilizar los aceites que se generan en los restaurantes de la zona, considerando las metodologías más eficientes de los resultados previamente analizados. Además, se explica el funcionamiento y las operaciones que se lleven a cabo.

\section{RECONOCIMIENTO}

A José Zarate Vega y Mirtha Gamarra Luján, mis padres. A Oscar Cubas y Flor Gamarra, en memoria suya.

\section{REFERENCIAS}

[1] Agencia de Protección Ambiental de Estados Unidos (2021). Desarrollo no convencional de petróleo y gas natural. https://www.epa.gov/uog

[2] M, Físico. Economía. España. Editex, 2019.

[3] Telesurtv.net. (2021). Conoce el estado del Protocolo de Kioto sobre cambio climático.https://www.telesurtv.net/news/protocolo-kioto-cambioclimatico-estado-actual-20210215-0082.html

[4] Ministerio Del Ambiente (2018). Ley Marco sobre Cambio Climático. https://sinia.minam.gob.pe/documentos/ley-marco-cambio-climatico

[5] J. Rocha, P. Salazar y J. Medrano. "Producción e impacto del biodiesel: una revisión”. INNOVA Research Journal, vol.2, no. 7, pp. 59-76. https://doi.org/10.33890/innova.v2.n7.2017.229

[6] Organización de las Naciones Unidas para la Educación, la Ciencia y la Cultura (2017). Programa Mundial de Evaluación de los Recursos Hídricos (WWAP) Hecho 22: Agua y los biocombustibles. http://www.unesco.org/new/es/natural-

sciences/environment/water/wwap/facts-and-figures/all-facts-wwdr3/fact22-water-biofuels/

[7] Keating, D. (2018). Biocombustibles: ¿buenos o malos para el medio ambiente? https://www.dw.com/es/biocombustibles-buenos-o-malos-parael-medio-ambiente/a-44396078

[8] A. Quimis. "Estudio del aceite utilizado en locales de comida rápida, para su aprovechamiento en biodiésel en el casco urbano del cantón Jipijapa". Tesis de licenciatura, Universidad Estatal del sur de Manabí, Ecuador, $2019 . \quad$ Repositorio Digital UNESUM. http://repositorio.unesum.edu.ec/handle/53000/2393

[9] A. Bardales y S. Casas. "Evaluación de alternativas de financiamiento para implementar una planta procesadora de biodiésel a partir de aceites usados y grasa animal, en la provincia de Chiclayo 2017". Tesis de licenciatura, Universidad Católica Santo Toribio de Mogrovejo, Lambayeque, Perú ,2018. https://tesis.usat.edu.pe/handle/20.500.12423/2156

[10] El Comercio. (2019). Solicitan a Municipalidad de Lima acciones sobre desecho de aceite quemado. https://elcomercio.pe/lima/sucesos/ministeriopublico-solicita-municipalidad-lima-acciones-desecho-aceite-quemadorestaurantes-noticia-643620-noticia/

[11]C. Duhram. (2020). Tirar grasa o aceites por el fregadero puede causar daños al sistema de alcantarillado. https://lanoticia.com/tirar-grasa-oaceites-por-el-fregadero-puede-causar-danos-al-sistema-de-alcantarillado/

[12] El Comercio. (2017). Reciclaje de aceite usado: estos son los puntos de acopio para entregar botellas. Recuperado de https://elcomercio.pe/lima/sucesos/reciclaje-aceite-usado-puntos-acopiobotellas-miraflores-444534-noticia/

[13]Energías renovables. (2021). El aceite de cocina usado procedente de China es la principal materia prima del biodiésel que se consume en España. https://www.energias-renovables.com/biocarburantes/el-aceite-de-cocinausado-procedente-de-20210215

[14]R. Pareja. (2020). ¿Son los biocombustibles realmente ecológicos? https://www.caranddriver.com/es/coches/planetamotor/a33260367/biocombustibles-ecologicos-mito-o-realidad/

[15] Gestión. (2021). Reclamo de Argentina podría dejar a 48,000 familias sin trabajo, advirtió Perú Palmas. Recuperado de https://gestion.pe/economia/reclamo-de-argentina-podria-dejar-a-48000familias-sin-trabajo-advirtio-peru-palmas-nndc-noticia/?ref=gesr

[16] El Comercio. (2017). Reciclaje de aceite usado: estos son los puntos de acopio para entregar botellas. Recuperado de https://elcomercio.pe/lima/sucesos/reciclaje-aceite-usado-puntos-acopiobotellas-miraflores-444534-noticia/

[17]La República. (2019). ¿Qué sucede cuando arrojas grasas y residuos sólidos al alcantarillado? Recuperado de https://larepublica.pe/sociedad/2019/11/04/que-sucede-cuando-arrojasgrasas-y-residuos-solidos-al-alcantarillado-agua-potable-consorcio-limanorte-lote-3/ 
[18]El Peruano. (2017). Declaran obligatoriedad de contar con trampas de grasa en los establecimientos comerciales del distrito. https://busquedas.elperuano.pe/normaslegales/declaran-obligatoriedad-decontar-con-trampas-de-grasa-en-lo-ordenanza-no-379-mds-1539785-1/

[19] Organización de las Naciones Unidas. (2019). Objetivos de Desarrollo Sostenible. https://www.un.org/sustainabledevelopment/es/objetivos-dedesarrollo-sostenible/

[20]B. Suazo. "Economía Circular en Chile: Alcances, problemas y desafíos en la gestión de la ley REP". Tesis de licenciatura, Universidad de chile, 2017. http://repositorio.uchile.cl/handle/2250/146815

[21]G. Gonzales. (2015). Valorización energética de aceites vegetales desechados para la producción catalítica heterogénea de biodiésel. Universidad de Chile. Tesis de licenciatura. http://repositorio.uchile.cl/bitstream/handle/2250/133214/Valorizacionenergetica-de-aceites-vegetales-desechados-para-la-produccioncata....pdf? sequence $=1$ \&isAllowed $=y$

[22]L, Fernández y M, Gutiérrez. (2013). Bienestar Social, Económico y Ambiental para las Presentes y Futuras Generaciones. https://scielo.conicyt.cl/pdf/infotec/v24n2/art13.pdf

[23]V. G. Tacias, A. Rosales y B. Torrestiana. Evaluación y caracterización de grasas y aceites residuales de cocina para la producción de biodiésel: un caso de estudio. Revista internacional de contaminación ambiental, 32(3), 303-313, mayo 2015 doi: https://dx.doi.org/10.20937/RICA.2016.32.03.05

[24]A. M. Baca. "Determinación de las características fisicoquímicas del aceite residual de frituras de los establecimientos de comida del mercado municipal de Huamachuco". Tesis de licenciatura. UNT, La Libertad, Perú, 2019. http://dspace.unitru.edu.pe/handle/UNITRU/13350

[25]K. Castillo. "Caracterización teórica de parámetros del biodiésel y estudio de algunas de sus emisiones". Tesis de licenciatura, Universidad Politécnica de Madrid. España, 2018. Repositorio Universidad Politécnica de Madrid. http://oa.upm.es/53357/1/TFG_KEVIN_CASTILLO_FERNANDEZ.pdf

[26]J. Herrera. (2008). Caracterización y aprovechamiento del aceite residual de frituras para la obtención de un combustible (biodiesel). https://core.ac.uk/reader/71395470

[27]G. E. Briones et al. Aplicaciones de sales inorgánicas en el tratamiento de aguas residuales industriales procedente de la refinación de aceites y grasas. Revista Colón Ciencias, Tecnología y Negocios ,2 (7), mayo 2020. http://portal.amelica.org/ameli/jatsRepo/215/2151282005/html/

[28]M. Villadiego, Y. Roa, y L. Benítez. Esterificación y transesterificación de aceites residuales para obtener biodiesel. Luna Azul, mayo 2015. doi: 10.17151/luaz.2015.40.3

[29]L. Delta. Química orgánica II con enfoque en alimentos. Delta \& Jules. España, 2020.

[30]P, Atkins. y L, Jones. Principios de química. Los caminos del descubrimiento. Médica Panamericana. 3ra ed. Argentina, 2012.

[31]H, Kessler. Libro de bolsillo de física. Ingeniería de alimentos y bioprocesos. 1996

[32]P, Vértiz. (2009). Análisis técnico y económico sobre producción, almacenamiento y transporte de biodiesel en Perú. https://pirhua.udep.edu.pe/bitstream/handle/11042/1279/IME_141.pdf?se quence $=1 \&$ is 1 llowed $=\mathrm{y}$

[33]J, Rodríguez et al. (2016). Determinación del índice de acidez y acidez total de cinco mayonesas. Investigación y Desarrollo en Ciencia y Tecnología de Alimentos. http://www.fcb.uanl.mx/IDCyTA/files/volume1/2/10/146.pdf

[34]F. Costa, P. Castro y E. Cortijo. (2008). Manual de construcción y de reactor para la producción de biodiésel a pequeña escala. Soluciones Prácticas - ITDG. Perú, 2008.

[35]H. Ramírez, H. Arteaga y R. Sich. (2012). Optimización del proceso de obtención de biodiesel a partir de colza silvestre (Brassica Campestris). Scientia Agropecuaria. 35 - 44

[36]L. López, J. Bocanegra y D. Malagón- Romero. Obtención de biodiésel por transesterificación de aceite de cocina usado. Ingeniería y Universidad, 19(1), 7-24, junio 2015. doi: 10.11144/Javeriana.iyu19-1.sprq

[37]C. Rivera P. Rivera y J. Rizo. (2015). Desarrollo de un método analítico alternativo para la determinación del porcentaje de humedad y materia volátil en aceite vegetal de uso comestible, periodo de marzo-julio del 2015.http://riul.unanleon.edu.ni:8080/jspui/bitstream/123456789/4224/1/2 29228.pdfb

[38]Federación Española Para el Desarrollo de Nutrición Animal. (2012). MIU (Moisture, impurities, Unsaponifiable) Impurezas Insolubles. http://www.fundacionfedna.org/tecnicas_de_analisis/miu-moistureimpurities-unsaponifiable-impurezas-insolubles

[39]M. Santana et al. Composición química y calidad de la grasa contenida en frituras de maíz elaboradas y consumidas en Navojoa, estado de Sonora, México, julio 2019. DOI: 10.17533/udea.penh.v21n1a02

[40]H. R. Sanaguano. "Conversión de los aceites residuales de la industria de alimentos en biodiésel". Tesis doctoral, UNMSN, Lima, Perú, 2018. https://cybertesis.unmsm.edu.pe/

[41]M. Toledo. "Obtención de Biodiesel a partir de aceites vegetales usados", Tesis de licenciatura, UNC, Lima, Perú, 2012. http://repositorio.unac.edu.pe/handle/UNAC/2065

[42]D. L. Gallegos y E. J. Llanos. "Simulación del proceso para la obtención de biodiesel a partir de aceites usados usando datos obtenidos a nivel de laboratorio". Tesis de licenciatura, UNT, La Libertad, Perú, 2013. https://dspace.unitru.edu.pe/handle/UNITRU/3449

[43]C. J. De la Cruz y C. A. Trujillo. "Obtención de biodiésel a partir de aceite comestible residual del comedor de la UNAC". Tesis de licenciatura, UNAC, Lima, Perú, 2017. http://repositorio.unac.edu.pe/handle/20.500.12952/3595

[44]L, Zavaleta y J. Suavo. "Obtención de Biodiésel por transesterificación Alcalina a partor de Aceites Vegetales Residual en Lima", TECNIA, vol. 26, 107-114, junio 2016. https://doi.org/10.21754/tecnia.v26i1.116

[45]B. Castillo. "Aprovechamiento de los desechos de aceites vegetales generados por el comedor universitario de la U.N.T. para la producción de biodiesel". Tesis de licenciatura, UNT, La Libertad, Perú, 2017. https://dspace.unitru.edu.pe/handle/UNITRU/8955

[46]M. Alva y P. Cipra. "Estudio comparativo de los biodiesel, obtenido a partir de metanol y etanol y su adaptación a escala piloto". Tesis de licenciatura, UNT, La Libertad, 2015. https://dspace.unitru.edu.pe/handle/UNITRU/ 1833

[47]J. C. Cedrón et al. "Análisis de biodiésel preparado a partir de residuos de aceite doméstico, mediante RMN", Scielo, vol.80, pp.3-8, febrero, 2014. http://www.scielo.org.pe/scielo.php?script=sci_arttext\&pid=S1810634X2014000100002

[48]K. V. Rodríguez y L. M. Villanueva. "Producción de Biodiesel a partir de Aceite Vegetal usado en Fritura por medio de Transesterificación de Metanol". Tesis de licenciatura, UNT, Lima, Perú, 2011. https://dspace.unitru.edu.pe/handle/UNITRU/3356

[49]M. Cabrera. "Obtención de biodiesel a partir de aceite residual comestible utilizando alúmina como catalizador", Tesis de licenciatura, UNJBG, Lima, Perú, 2017. http://repositorio.unjbg.edu.pe/handle/UNJBG/1574

[50]J. Rodríguez. Evaluación del rendimiento de obtención de biodiesel mediante el proceso de transesterificación de aceite de soja usado por el método de Superficie de respuesta. Tesis de licenciatura, UPU, Lima, Perú, 2018. https://repositorio.upeu.edu.pe/handle/20.500.12840/1381

[51]M. Osorio. "Mejora de Procesos para optimizar los volúmenes de obtención de glicerina y biodiésel en laboratorio a partir de aceite vegetal reciclado en la UCV-ATE, 2018". Tesis de licenciatura, UCV, Lima, Perú, 2018. https://repositorio.ucv.edu.pe/handle/20.500.12692/24359

[52]M. Santiago. Preparación a escala planta piloto y estudio comparativo de los biodiesel obtenidos a partir de metanol y etanol. Tesis de licenciatura, UNAC, Lima, Perú, 2008. http://repositorio.unac.edu.pe/handle/20.500.12952/ 408

[53]J. Salazar y F. Valle. "Optimización de la concentración de etanol e hidróxido de potasio en el rendimiento y características fisicoquímicas de biodiesel de aceite de cocina usado obtenido en un prototipo automatizado". Tesis de licenciatura, UNT, Trujillo, Perú, 2018. http://www.dspace.unitru.edu.pe/handle/UNITRU/11898

[54] J. Paredes y M. Vidal. "Diseño y construcción de una planta de producción de Biodiesel a partir de aceite vegetal reciclado". Tesis de licenciatura, USFQ, Ecuador, 2017. https://repositorio.usfq.edu.ec/handle/23000/5994

[55]A. Gil. "Estudio de viabilidad, diseño y montaje de una planta de biocombustible para el autoabastecimiento de una comunidad rural en un país en desarrollo". Tesis de licenciatura, UPM. España, 2015. http://oa.upm.es/43847

[56]D.Y Leung, et al. "Una revisión sobre la producción de biodiésel mediante transesterificación catalizada". Applied Energy, vol 87 pp 1083-1095, abril, 2010.https://www.sciencedirect.com/science/article/abs/pii/S0306261909 004346

[57]A. K. Varty y S. C. Lishawa. (2008). Haciendo biodiesel de aceite vegetal usado. https://greenteacher.com/article\%20files/haciendobiodiesel. pdf. 